
\title{
$\begin{array}{ll}\text { Research Square } & \begin{array}{l}\text { Preprints are preliminary reports that have not undergone peer review. } \\ \text { They should not be considered conclusive, used to inform clinical practice, } \\ \text { or referenced by the media as validated information. }\end{array}\end{array}$ \\ Optimal Discrete-Time Sliding-Mode Control Based on Recurrent Neural Network: A Singular Value Approach
}

\section{Hamid Toshani}

Iran University of Science and Technology

Mohammad Farrokhi ( $\nabla$ farrokhi@iust.ac.ir)

Iran University of Science and Technology https://orcid.org/0000-0002-8431-4650

\section{Research Article}

Keywords: Discrete sliding-mode control, Projection recurrent neural network, Optimality, Singular value approach, Control limit, Uncertainty

Posted Date: June 22nd, 2021

DOl: https://doi.org/10.21203/rs.3.rs-631625/v1

License: (c) This work is licensed under a Creative Commons Attribution 4.0 International License.

Read Full License 


\title{
Optimal discrete-time sliding-mode control based on recurrent neural network: a singular value approach
}

\author{
Hamid Toshani ${ }^{1}$, Mohammad Farrokhi ${ }^{1,2}{ }^{*}$ \\ ${ }^{1}$ School of Electrical Engineering, Iran University of Science and Technology, Narmak, Farjam St., Tehran 16846, Iran \\ ${ }^{2}$ Center of Excellence for Modeling and Control of Complex Systems, Iran University of Science and Technology, \\ Narmak, Farjam St., Tehran 16846, Iran \\ *farrokhi@iust.ac.ir
}

\begin{abstract}
In this paper, a strategy involving the combination of optimal discrete-time sliding-mode control and recurrent neural networks is proposed for a class of uncertain discrete-time linear systems. First, a performance index based on the reaching law and the control signal is defined. Then, the constrained quadratic programming problem is formulated considering the limitations on the control signal as the static constraint. The dynamic and algebraic model of the neural network is derived based on the optimization conditions of the quadratic problem and their relationship with the projection theory. The proposed method prevents the chattering by selecting proper parameters of the twisting reaching law. The convergence of the neural network is analysed using the Lyapunov stability theory. A singular-value-based analysis is employed for robustness of the proposed method. The stability conditions of the discrete-time closedloop system is analysed by studying eigenvalues of the closed-loop matrix using the singular value approach. The performance of the proposed algorithm is assessed in simulated example in terms of chattering elimination, solution feasibility, and encountering uncertainties and is compared with the recently proposed DSMC methods in the literature.
\end{abstract}

Keyword Discrete sliding-mode control; Projection recurrent neural network; Optimality; Singular value approach; Control limit; Uncertainty

\section{Introduction}

The variable-structure control systems form a significant subcategory of nonlinear control systems due to their simple design, satisfactory accuracy, quick response, high generalizability and their capability to maintain robustness of the closed-loop system in the face of structured and unstructured uncertainties.

In systems based on data sampling, direct application of continuous-time control involves considerable difficulty including sampling limitations, significant increase in chattering amplitude, discretization error and possibility of instability in the closed-loop system (Ma et al. 2016). In fact, implementing a continuous-time control system using digital computers requires proper sampling time in order to overcome chattering and at the same time involves instability as a result of increase in the control gains. The influence of sampling time has been studied by Furuta; it has been shown that chattering elimination requires a considerable fast sampler (2019). The robustness of a closed-loop system in the presence of an analogto-digital converter and its effect on computing the control law is also investigated by Amini et al. (2017).

With the development of digital processors and the possibility of realizing control system hardware based on sampled signals, Discrete-time Sliding-Mode Control (DSMC) has come to numerous researchers' attention. Direct utilization of DSMC has plenty of advantages including high robustness, lower sensitiveness to temperature variations, less mechanical depreciation, and lower costs (Yan 2018). DSMC theory came about in 1980 along with the development of a discrete-time model for the reaching law (Gao et al. 1995). Moreover, the effect of the appropriate sampling range on the chattering amplitude and the choice of the upper bound for the sampling frequency has been examined by Furuta (2019). Since an approximation of the reaching law is considered in discretetime systems, the system states do not perfectly converge on the sliding surface. As a result, an idea named Discrete-time Quasi Sliding-Mode Control (DQSMC) has emerged (Bartoszewicz 1998) and has been used for an underwater robot subject to parametric uncertainties (Lee et al. 1999). This method is assessed in the presence of uncertainties, with appropriate strategy to reduce the domain bandwidth of the quasi slidingmode (Ma et al. 2016).

An unwanted phenomenon in the field of SMC is the chattering, which causes fluctuations in the control signals or the system states. This can wear out the actuators and degrade performance of the closed-loop system. Chattering is a result of delay in the switching along with the high-frequency components in the un-modeled dynamics of actuators. Various strategies have been suggested in order to deal with this problem. An approximate recurrent switching function based on real-time estimation of the perturbation information for a class of linear systems with parametric changes and external perturbations by Milosavljević et al. (2016) recursively estimating the nonlinear disturbance term in real-time has been proposed by Du et al. (2016), where the switching term is replaced with a continuous function. In addition, a continuous function as the switching term to eliminate chattering that depends on the real-time value of the sliding surface and the upper bound of the uncertainty has been used by Golo and 
Milosavljević (2000). Moreover, studying the boundedness of the sliding surface by employing generalized reaching law (Chakrabarty and Bandyopadhyay 2015), and investigating the boundedness of a fast terminal sliding-mode control (Xiong et al. 2016) are other issues related to chattering.

One of the most important ways to counter chattering is developing second-order sliding-mode control strategy. Methods based on the second derivative of the sliding surface and those based on the twisting algorithm may be named among these strategies. The effects of chattering using sliding condition, generalized with the aid of finite-difference method has been investigated in the literature, where the closed-loop stability has been examined based on the ultimate boundedness criterion in the presence of disturbance (Sharma and Janardhanan 2017). In addition, an implicit time-warping algorithm, rather than an explicit one has been proposed in the literature (Huber et al. 2014). It has been shown that the implicit method is significantly more capable of encountering the effects of chattering in the control signal. This has been done with the aid of convex analysis and variational inequality theory, where specialty software package is required to compute the control signal. Discrete-time second-order sliding-mode control strategies have been utilized in applications such as electrohydraulic actuator (Zhou et al. 2017) and piezoelectric positioning control (Xu and Cao 2017).

To overcome uncertainties in the field of DSMC, numerous studies have been conducted. The Linear Matrix Inequality (LMI) approach has been used for a class of uncertain linear adaptive systems (Argha et al. 2016). Moreover, conditions for the boundedness of closed-loop stability have been studied by estimating noise via LMI. It has been shown that by selecting output with relative degree of two for a class of discrete-time uncertain LTI systems, one may achieve better robustness and finite-time stability compared with the output with relative degree of one (Chakrabarty and Bartoszewicz 2016). Countering repetitive scalar uncertainties for a class of switching discrete-time systems has been considered in the literature, where the $H_{\infty}$-gain method has been employed to optimize the transient response while guaranteeing closed-loop exponential stability (Su et al. 2019).

In the majority of the aforementioned studies, problems such as optimization in the sliding surface, minimum control effort, and feasibility of the control space have not been examined. In this manuscript, a constrained optimal slidingmode control approach based on recurrent neural networks (RNNs) is proposed for a class of uncertain discrete-time systems. The RNN performs based on the projection theory, which represents a first-order dynamic model with a parallel structure and piece-wise linear activation functions. The employed projection RNN (PRNN) determines the SMC laws at every instant as an optimizer. These networks have been developed before to solve various kinds of constrained optimization problems including linear programming (Liu et al. 2010), second-order programming (Yan 2014; Feng et al. 2017; Miao et al. 2014), nonlinear programming (Liu and Wang 2013; Xia and Wang 2005) and min-max optimization problems (Liu and Wang 2015).

The design criteria for the PRNNs are derived based on the Karush-Kuhn-Tukher optimality conditions and the variational inequality theory (Kinderlehrer and Stampacchia 1980). These networks are structurally simple and are suitable for online applications due to their fast performance. High applicability and generalizability along with strong convergence analysis capability are among other advantages of the PRNNs.

To derive the dynamic model of the neural network, a control performance index is defined and formulated based on the control signals as the optimization variables. Then, the optimization problem is reformulated as a quadratic programming $(\mathrm{QP})$ problem. To guarantee the closed-loop system stability in the presence of uncertainties, the singular value approach (a suitable method for the discrete-time systems) will be used. The advantages of the proposed approach may be summarized as follows:

- Feasibility of the control space with the consideration of structural constraints of the actuators

- Optimal SMC by defining a performance index based on the minimum control effort

- Singularity avoidance

- Chattering avoidance by deriving the control law using the twisting algorithm

- Robustness against parametric and non-parametric uncertainties

- Possibility of considering the constraints of the output and system states

- Rapid convergence of the optimal control problem by employing a neuro-optimizer

- Guaranteeing closed-loop stability in the presence of uncertainties

This paper is organized as follows. Section 2 presents the problem statement involving constrained optimal sliding-model control with the definition of a performance index and control constraints. Section 3 provides structure of the neuro optimizer. Section 4 investigates the closed-loop system stability using singular value approach. In Section 5, simulation results and performance assessment of the proposed algorithm will be presented. Section 6 concludes the paper.

\section{Problem statement}

Consider the following discrete-time single-input singleoutput dynamic system:

$$
\begin{aligned}
& \mathbf{x}(k+1)=\mathbf{A} \mathbf{x}(k)+\mathbf{b} u(k)+\mathbf{w}(k) \\
& y(k)=\mathbf{c} \mathbf{x}(k)
\end{aligned}
$$

where $\mathbf{x} \in R^{n}, y \in R$ and $u \in R$ are the state vector, the output and the input signals, respectively, $\mathbf{w} \in R^{n}$ denotes the vector of uncertainties including the modeling error and the external disturbances. Moreover, $\mathbf{A} \in R^{n \times n}$ is the time-invariant state matrix and $\mathbf{b} \in R^{n}$ and $\mathbf{c}^{T} \in R^{n}$ are the input and output vectors, respectively. The variable $k$ denotes the time sample. It is assumed that $k t_{s}:=k$, where $t_{s}$ is the sampling time.

It is worth mentioning that the discrete-time model presented in (1) may be developed for any nonlinear discretetime system that is separable into linear and nonlinear parts. In other words, the nonlinear terms of the system dynamics can be considered as part of the uncertainty vector $\mathbf{w}$. 
Assumption 1 The upper bound of the uncertainty vector $\mathbf{w}$ is known. In other words, $\|\mathbf{w}\| \leq \Gamma$.

The main purpose of the control algorithm is tracking the reference input $r_{d}(k) \in R$ with minimum error and quick response. Therefore, the error signal is defined as

$e(k)=y(k)-r_{d}(k)$.

Consider the sliding surface as

$s(k)=\alpha e(k)$,

where $\alpha>0$ is the design parameter. In (3), it is assumed that the relative degree of the system in (1) is equal to one. It means that $\mathbf{c b} \neq 0$.

In the proposed algorithm, the aim is to track the desired inputs and to insure the internal stability of the closed-loop system as well. The main reason to choose the sliding surface as described in (3) is to perform the tracking problem accurately. Moreover, the singular value approach is used to guarantee the closed-loop stability. In this regard, the conditions for designing the parameters to insure internal stability, to overcome the model uncertainties, and to obtain the minimum tracking error will be derived.

In this paper, the existence condition of the sliding mode (i.e., the reaching law) for the discrete-time dynamic system is considered as follows (Gao et al. 1995):

$s(k+1)=a_{1} s(k)-a_{2} \operatorname{sgn}(s(k))$,

where $a_{1}=\left(1-\lambda_{1} t_{s}\right)$ and $a_{2}=\lambda_{2} t_{s}$, in which $\lambda_{1}$ and $\lambda_{2}$ are the design parameters.

For the system states to converge to the sliding surface from any initial condition and remain on it, the relation $1-\lambda_{1} t_{s}>0$ must hold. This means that the existence of the sliding mode fully depends on the sampling time.

The optimal SMC law will be derived by defining the following performance index:

$$
J(k)=\frac{1}{2} \eta\left[s(k+1)-a_{1} s(k)+a_{2} \operatorname{sgn}(s(k))\right]^{2}+\frac{1}{2} \mu u^{2}(k),
$$

where $\eta$ and $\mu$ are positive scalars which are determined based on the robust stability analysis of the closed-loop system.

Hence, the optimization algorithm comprises of two goals: 1) minimization of the sliding-mode variation rate and 2) minimization of the control effort.

Remark 1 The minimum rate of the sliding-surface variations is in accordance with the principal of the SMC law. By minimizing the first term of the performance index in (5), i.e.

$$
\frac{1}{2} \eta\left[s(k+1)-a_{1} s(k)+a_{2} \operatorname{sgn}(s(k))\right]^{2}
$$

relation $\lim s(k+1)-a_{1} s(k)+a_{2} \operatorname{sgn}(s(k))=0$ holds, which means that the reaching law in (4) will be satisfied. Based on (4), in order to extract the equivalent control law, the variation rate of the sliding surface $(s(k+1)-s(k))$ must be zero; while the other terms of the reaching law are used to improve the convergence speed of the states $\left(-\lambda_{1} s(k)\right)$ and to overcome the model uncertainty $\left(a_{2} \operatorname{sgn}(s(k))\right)$.

Parameter $\mu>0$ in the cost function (5), in addition to adjusting the importance of the control signal amplitude, plays a significant role in the numerical stability of the neural optimizer algorithm. This will be discussed in Section 4.

In (5), the control signal $u$ is recognized as the optimization variable. Therefore, the variation rate of the sliding-surface has to be formulated based on the control signal. In other words, it can be written

$s(k+1)=\alpha e(k+1)=\alpha y(k+1)-\alpha r_{d}(k+1)$.

Using the output equation in (1), it gives

$s(k+1)=\alpha \mathbf{c x}(k+1)-\alpha r_{d}(k+1)$.

Ignoring the uncertainty term $\mathbf{w}(k)$, it yields

$s(k+1)=\alpha \mathbf{c A x}(k)+\alpha \mathbf{c b} u(k)-\alpha r_{d}(k+1)$.

Remark 2 The effects of the model uncertainties will be considered in stability analysis of the closed-loop system using the singular value approach (Section 4).

Therefore, the performance index can be reformulated as

$$
\begin{aligned}
J(k)=\frac{1}{2} \eta[ & \alpha \mathbf{c A x}(k)+\alpha \mathbf{c b} u(k)-\alpha r_{d}(k+1) \\
& \left.-a_{1} s(k)+a_{2} \operatorname{sgn}(s(k))\right]^{2}+\frac{1}{2} \mu u^{2}(k) .
\end{aligned}
$$

Or in simple form

$$
J(k)=\frac{1}{2} Q u^{2}(k)+h(k) u(k)+J_{\min }(k),
$$

where

$$
\begin{aligned}
& Q=\eta(\alpha \mathbf{c b})^{2}+\mu, \\
& h(k)=\eta \alpha \mathbf{c b}\left(\alpha \mathbf{c}\left(\mathbf{A}-a_{1} \mathbf{I}_{n \times n}\right) \mathbf{x}(k)\right) \\
& +\eta \alpha \mathbf{c b}\left[-\alpha r_{d}(k+1)+a_{2} \operatorname{sgn}(s(k))-a_{1} \alpha r_{d}(k)\right], \\
& J_{\text {min }}(k)=\frac{1}{2} \eta\left[\alpha \mathbf{c A x}(k)-\alpha r_{d}(k+1)-a_{1} s(k)\right]^{2} .
\end{aligned}
$$

$J_{\min }$ in (10) has no explicit relationship with the optimization variable $(u)$. Hence, the gradient of $J_{\min }$ with respect to the control signal is zero. Thus, this term does not affect the structure of the neuro-optimizer and can be omitted in the optimization problem.

In this paper, the physical limits of the actuator $u_{\min } \leq u(k) \leq u_{\max }$ is considered as the static constraints, where $u_{\min }$ and $u_{\max }$ are the lower and upper bounds of the control signal, respectively.

Therefore, the optimal sliding-mode control problem can be expressed as the following constrained optimization problem:

$$
\begin{aligned}
& \min _{u(k)} J(k)=\frac{1}{2} Q u^{2}(k)+h(k) u(k) \\
& \text { s.t. } \quad u_{\min } \leq u(k) \leq u_{\max },
\end{aligned}
$$


which is in QP form and denotes a constrained and convex optimization space where $h(k)$ only gives an offset to the cost function.

Remark 3 The initial performance index in (5) does not necessarily represent a convex optimization problem, while by formulating it in terms of the control signal and defining appropriately the sliding surface, a convex QP problem is formed in (11).

\section{Neuro-Optimizer}

In order to solve the constrained QP problem subject to static constraint, projection theory, which rooted in the variational inequality for solving constrained optimization problems, is employed in this manuscript.

The constrained QP problem in (11) can be transformed into a dual problem as follows (Liu and Wang 2006):

$$
\begin{array}{ll}
\max _{u(k)} J(k)=-\frac{1}{2} Q u^{2}(k)+u_{\text {min }} \omega(k)-u_{\text {max }} \varphi(k) \\
\text { s.t. } \quad Q u(k)+h(k)-(\omega(k)-\varphi(k))=0,
\end{array}
$$

where $\omega \in R$ and $\varphi \in R$ are decision variables.

By defining a new variable as $\psi(k):=\omega(k)-\varphi(k)$, the equality constraint in (13) can be rewritten as follows:

$Q u(k)+h(k)-\psi(k)=0$.

Since $\psi$ acts as a decision variable, it can be found that if $\psi(k)=0$, then $u(k)=-Q^{-1} h(k)$ and based on (11) the constraints are satisfied. On the other hand, if $\psi(k)>0$, then $u(k)=Q^{-1}(\psi(k)-h(k))$ and according to (11) $u(k)=u_{\min }$. Moreover, when $\psi(k)<0$, then it yields that $u(k)=u_{\max }$. In fact, the decision variable $\psi$ determines (based on the active set method in the constrained optimization problems) whether the optimization variable converges inside the optimization space or on the boundaries.

Based on the relation between $\psi$ and the constraint in (11), the projection equation can be expressed as follows:

$$
\operatorname{Pr}_{\Omega}\{u(k)-\psi(k)\}=u(k)
$$

where $\operatorname{Pr}_{\Omega}\{\cdot\}$ denotes the projection operator and is described as a piecewise linear function as

$$
\operatorname{Pr}_{\Omega}\{z\}=\left\{\begin{array}{lll}
u_{\min } & \text { if } \quad z<u_{\min } \\
u_{\max } & \text { if } \quad z>u_{\max } \\
z & \text { if } \quad u_{\min } \leq z \leq u_{\max }
\end{array} .\right.
$$

Moreover, $\Omega$ is the constrained space; i.e., $\Omega=\left\{u(k) \mid u_{\min } \leq u(k) \leq u_{\max }\right\}$. Eq. (14) can be related to the following projection theorem for solving the optimization problem (Xia and Wang 1998):

$\operatorname{Pr}_{\Omega}\left\{\mathbf{x}^{*}-U\left(\mathbf{x}^{*}\right)\right\}=\mathbf{x}^{*}$, where $U(\mathbf{x})$ is the gradient of the cost function and $\mathbf{x}^{*}$ is the optimal point. In general, the projection operator $\operatorname{Pr}_{\Omega}\{\cdot\}$ is described as follows:

$\operatorname{Pr}_{\Omega}\left\{\mathbf{x}^{*}\right\}=\arg \min _{\chi \in \Omega}\left\|\mathbf{x}^{*}-\chi\right\|$.

Based on (17), the aim of the projection theory is to find $\chi \in \Omega$ such that it has the minimum distance from the optimal point $\mathbf{x}^{*}$. The structure of the PRNN that guarantees (14) can be described as

$\psi(k+1)=\psi(k)+\zeta t_{s}\left(\operatorname{Pr}_{\Omega}\{u(k)-\psi(k)\}-u(k)\right)$,

where $\zeta$ is the learning rate of the neural network.

Based on (14), the control law can be derived as the output equation of the PRNN as

$u(k)=Q^{-1}(\psi(k)-h(k))$.

Equations (18) and (19) represent a recurrent neural network with parallel configuration and piecewise-continuous activation functions. Availability of the output equation (19) is required for analysing stability of the closed-loop system. The PRNN structure generates the control law at every instant with the aid of equation (19) and by realizing the optimization constraints in the dynamic equation (19). Moreover, the number of the PRNN neurons is equal to the number of constraints.

The block diagram of the PRNN is shown in Fig. 1 . According to Fig. 1, piecewise functions act as the activation operators for the input data. The PRNN structure has been inspired by Hopfield NNs, in which the states are converged to NN's equilibrium point. Therefore, unlike the multi-layered feedforward neural networks, no weight adaptation is required. By selecting proper values for the designing parameters $\eta, \mu, \alpha$ and $\lambda_{1,2}$ a chattering-free SMC law with minimum effort and minimum tracking error for the closed-loop system can be obtained.

It should be noticed that the PRNN described in (18) and (19) is asymptotically stable, which is shown in Appendix.

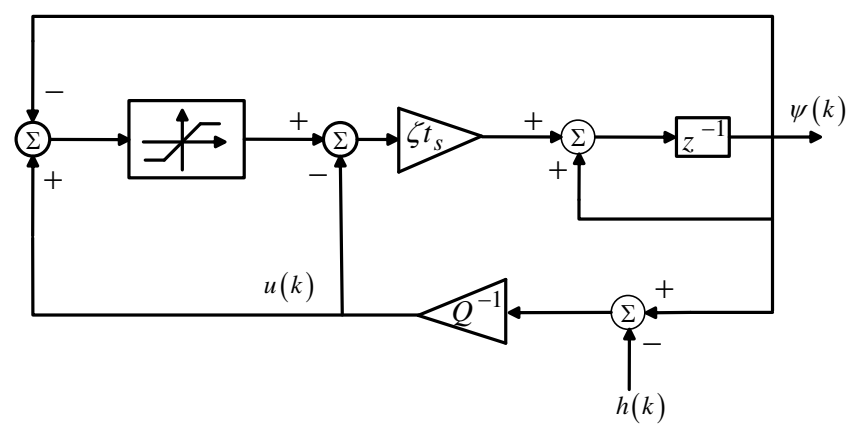

Fig. 1 Block diagram of PRNN for solving constrained QP

\section{Stability analysis of the closed-loop system}

In order to analyse stability of the closed-loop system in the presence of the model uncertainties, the singular value approach is used. Substituting control law (19) in (1) gives 


$$
\begin{aligned}
& \mathbf{x}(k+1)=\mathbf{A} \mathbf{x}(k)+\mathbf{b} Q^{-1}(\psi(k)-h(k))+\mathbf{w}(k) \\
& y(k)=\mathbf{c x}(k) .
\end{aligned}
$$

Based on (10) and after some manipulations

$$
\begin{aligned}
\mathbf{x}(k+1) & =\left(\mathbf{A}-\mathbf{b} Q^{-1}(\eta \alpha \mathbf{c b})\left(\alpha \mathbf{c}\left(\mathbf{A}-a_{1} \mathbf{I}_{n \times n}\right)\right)\right) \mathbf{x}(k) \\
& +\mathbf{b} Q^{-1}(\eta \alpha \mathbf{c b})\left(\alpha r_{d}(k+1)+a_{1} \alpha r_{d}(k)\right) \\
& -\mathbf{b} Q^{-1}(\eta \alpha \mathbf{c b}) a_{2} \operatorname{sgn}(s(k)) \\
& +\mathbf{b} Q^{-1} \psi(k)+\mathbf{w}(k) \\
y(k)= & \mathbf{c x}(k) .
\end{aligned}
$$

The error dynamic is defined as

$$
\begin{aligned}
e(k+1): & =\mathbf{c}\left(\mathbf{A}-\mathbf{b} Q^{-1}(\eta \alpha \mathbf{c b})\left(\alpha \mathbf{c}\left(\mathbf{A}-a_{1} \mathbf{I}_{n \times n}\right)\right)\right) \mathbf{x}(k) \\
& +\mathbf{c b} Q^{-1}(\eta \alpha \mathbf{c b})\left(\alpha r_{d}(k+1)+a_{1} \alpha r_{d}(k)\right) \\
& +\mathbf{c b} Q^{-1} \psi(k)-\mathbf{c b} Q^{-1}(\eta \alpha \mathbf{c b}) a_{2} \operatorname{sgn}(s(k)) \\
& +\mathbf{c} \mathbf{w}(k)-r_{d}(k+1),
\end{aligned}
$$

which can be expressed in the following general form:

$$
e(k+1)=\mathbf{c G} x(k)+\overline{\mathbf{d}}(k),
$$

where

$$
\begin{aligned}
& \mathbf{G}=\mathbf{A}- \mathbf{b} Q^{-1}(\eta \alpha \mathbf{c b})\left(\alpha \mathbf{c}\left(\mathbf{A}-a_{1} \mathbf{I}_{n \times n}\right)\right) \\
& \overline{\mathbf{d}}(k)=\mathbf{c b} Q^{-1}(\eta \alpha \mathbf{c b})\left(\alpha r_{d}(k+1)+a_{1} \alpha r_{d}(k)\right) \\
&-\mathbf{c b} Q^{-1}(\eta \alpha \mathbf{c b}) a_{2} \operatorname{sgn}(s(k)) \\
&+\mathbf{c b} Q^{-1} \psi(k)+\mathbf{c w}(k)-r_{d}(k+1) .
\end{aligned}
$$

It is assumed that the upper bound of vector $\overline{\mathbf{d}}(k)$ is known. That is, $\|\overline{\mathbf{d}}(k)\| \leq \bar{\Gamma}$.

Theorem 1 The closed-loop system in (21) is asymptotically stable, if parameters $\mu, \alpha, \eta, a_{1}$ and $a_{2}$ are determined such that

a) The following inequality is satisfied:

$$
Q^{-1} \eta \alpha^{2}(\mathbf{c b})>\frac{\rho_{1}}{\rho_{2} \rho_{3}} \text {. }
$$

b) Parameters are obtained such that

$$
\left\{\begin{array}{l}
\mathbf{c G} \mathbf{x}_{\infty}+\mathbf{c b} Q^{-1}(\eta \alpha \mathbf{c b})\left(\alpha\left(a_{1}+1\right) r_{d}\right)=r_{d} \\
\mathbf{c b} Q^{-1}(\eta \alpha \mathbf{c b}) a_{2}=\Gamma_{w}
\end{array} .\right.
$$

where $\sigma_{\max }(\cdot)$ and $\sigma_{\min }(\cdot)$ denote the maximum and minimum singular value, respectively, and $\rho_{1}=1-\left(1 / \sigma_{\max }(\mathbf{A})\right)$, $\rho_{2}=\sigma_{\min }\left(\mathbf{A}^{-1} \mathbf{b}\right)$ and $\rho_{3}=\sigma_{\min }\left(\mathbf{c}\left(\mathbf{A}-a_{1} \mathbf{I}_{n \times n}\right)\right)$.

Proof The condition for closed-loop stability is that the absolute value of the eigenvalues of $\mathbf{G}$ in (23) are less than one. Hence, it is sufficient that its largest eigenvalue becomes lower than one. In other words,

$\left|\lambda_{\max }\left(\mathbf{A}-\mathbf{b} Q^{-1}(\eta \alpha \mathbf{c b})\left(\alpha \mathbf{c}\left(\mathbf{A}-a_{1} \mathbf{I}_{n \times n}\right)\right)\right)\right|<1$, where $\lambda_{\max }(\cdot)$ denotes the largest eigenvalue of the corresponding matrix. Inequality (27) is equivalent to the following inequality (Maciejowski 1989):

$$
\left\|\mathbf{A}-\mathbf{b} Q^{-1}(\eta \alpha \mathbf{c b})\left(\alpha \mathbf{c}\left(\mathbf{A}-a_{1} \mathbf{I}_{n \times n}\right)\right)\right\|_{1}<\infty,
$$

where $\|\cdot\|_{1}$ denotes norm one. For an arbitrary matrix $\mathbf{M} \in R^{n \times n}$, the following relation between its minimum singular value $\sigma_{\min }(\mathbf{M})$, its maximum singular value $\sigma_{\max }(\mathbf{M})$ and its eigenvalue $\lambda_{i}(\mathbf{M})$ holds (Skogestad and Postlethwaite 2007):

$0 \leq \sigma_{\text {min }}(\mathbf{M}) \leq\left|\lambda_{i}(\mathbf{M})\right| \leq \sigma_{\text {max }}(\mathbf{M}), \forall i=1, \ldots, n$.

Therefore, inequality (27) can be reformed as

$$
\sigma_{\max }\left(\mathbf{A}-\mathbf{b} Q^{-1}(\eta \alpha \mathbf{c b})\left(\alpha \mathbf{c}\left(\mathbf{A}-a_{1} \mathbf{I}_{n \times n}\right)\right)\right)<1 .
$$

Using the following relation:

$$
\sigma_{\max }(\mathbf{M})=\frac{1}{\sigma_{\min }\left(\mathbf{M}^{-1}\right)} \text {. }
$$

Inequality (29) can be expressed as below:

$$
\sigma_{\min }\left(\left[\mathbf{A}-\mathbf{b} Q^{-1}(\eta \alpha \mathbf{c b})\left(\alpha \mathbf{c}\left(\mathbf{A}-a_{1} \mathbf{I}_{n \times n}\right)\right)\right]^{-1}\right)>1 .
$$

Some manipulation on (31) yields

$$
\sigma_{\min }\left(\left[I-\mathbf{A}^{-1} \mathbf{b} Q^{-1}(\eta \alpha \mathbf{c b})\left(\alpha \mathbf{c}\left(\mathbf{A}-a_{1} \mathbf{I}_{n \times n}\right)\right)\right]^{-1} \mathbf{A}^{-1}\right)>1 .
$$

Moreover, for arbitrary matrices $\mathbf{M} \in R^{n \times n}$ and $\mathbf{N} \in R^{n \times n}$ there exists

$\sigma_{\min }(\mathbf{M N}) \geq \sigma_{\min }(\mathbf{M}) \sigma_{\min }(\mathbf{N})$.

Using (33), (32) can be expressed as follows:

$$
\sigma_{\min }\left(\left[\mathbf{I}-\mathbf{A}^{-1} \mathbf{b} Q^{-1}(\eta \alpha \mathbf{c b})\left(\alpha \mathbf{c}\left(\mathbf{A}-a_{1} \mathbf{I}_{n \times n}\right)\right)\right]^{-1}\right) \sigma_{\min }\left(\mathbf{A}^{-1}\right)>1,
$$

or

$$
\sigma_{\min }\left(\left[\mathbf{I}-\mathbf{A}^{-1} \mathbf{b} Q^{-1}(\eta \alpha \mathbf{c b})\left(\alpha \mathbf{c}\left(\mathbf{A}-a_{1} \mathbf{I}_{n \times n}\right)\right)\right]^{-1}\right)>\frac{1}{\sigma_{\min }\left(\mathbf{A}^{-1}\right)} .
$$

Using (30), it yields

$$
\sigma_{\min }\left(\left[\mathbf{I}-\mathbf{A}^{-1} \mathbf{b} Q^{-1}(\eta \alpha \mathbf{c b})\left(\alpha \mathbf{c}\left(\mathbf{A}-a_{1} \mathbf{I}_{n \times n}\right)\right)\right]^{-1}\right)>\sigma_{\max }(\mathbf{A}) .
$$

By defining $\mathbf{H}:=\mathbf{A}^{-1} \mathbf{b} Q^{-1}(\eta \alpha \mathbf{c b})\left(\alpha \mathbf{c}\left(\mathbf{A}-a_{1} \mathbf{I}_{n \times n}\right)\right)$, (36) can be rewritten as follows:

$$
\sigma_{\min }\left([\mathbf{I}-\mathbf{H}]^{-1}\right)>\sigma_{\max }(\mathbf{A}) \text {. }
$$

For an arbitrary H, we have (Meyer 2000)

$$
[\mathbf{I}-\mathbf{H}]^{-1}=\mathbf{I}+\mathbf{H}+\mathbf{H}^{2}+\mathrm{L}=\sum_{k=0}^{\infty} \mathbf{H}^{k}
$$

Using (38), it yields

$$
\sigma_{\min }\left([\mathbf{I}-\mathbf{H}]^{-1}\right)=\sigma_{\min }\left(\sum_{k=0}^{\infty} \mathbf{H}^{k}\right),
$$


or

$$
\sigma_{\min }\left([\mathbf{I}-\mathbf{H}]^{-1}\right)=\sum_{k=0}^{\infty} \sigma_{\min }\left(\mathbf{H}^{k}\right)=1+\sigma_{\min }(\mathbf{H})+\sigma_{\min }\left(\mathbf{H}^{2}\right)+\mathrm{L} .
$$

Therefore, inequality (37) can be reformulated as

$$
1+\sigma_{\min }(\mathbf{H})+\sigma_{\min }\left(\mathbf{H}^{2}\right)+\mathrm{L}>\sigma_{\max }(\mathbf{A}) .
$$

Based on (33), we can conclude

$$
\sigma_{\min }\left(\mathbf{H}^{k}\right) \geq\left(\sigma_{\min }(\mathbf{H})\right)^{k}, k=2,3, \mathrm{~K} .
$$

Using (42), (41) can be reformulated as follows:

$$
1+\sigma_{\min }(\mathbf{H})+\left(\sigma_{\min }(\mathbf{H})\right)^{2}+\left(\sigma_{\min }(\mathbf{H})\right)^{3}+\mathrm{L}>\sigma_{\max }(\mathbf{A}) .
$$

If $\sigma_{\min }(\mathbf{H}) \geq 1$, inequality (43) is always true. If $\sigma_{\min }(\mathbf{H})<1$, using the following relation for an arbitrary $0<\gamma<1$

$$
\sum_{k=0}^{\infty} \gamma^{k}=\frac{1}{1-\gamma}
$$

we can conclude that

$\frac{1}{1-\sigma_{\min }(\mathbf{H})}>\sigma_{\max }(\mathbf{A})$,

or

$$
\sigma_{\min }(\mathbf{H})>1-\frac{1}{\sigma_{\max }(\mathbf{A})} .
$$

Substituting $\quad \mathbf{H}=\mathbf{A}^{-1} \mathbf{b} Q^{-1}(\eta \alpha \mathbf{c b})\left(\alpha \mathbf{c}\left(\mathbf{A}-a_{1} \mathbf{I}_{n \times n}\right)\right) \quad$ into yields

$$
\sigma_{\min }\left(\mathbf{A}^{-1} \mathbf{b} Q^{-1}(\eta \alpha \mathbf{c b})\left(\alpha \mathbf{c}\left(\mathbf{A}-a_{1} \mathbf{I}_{n \times n}\right)\right)\right)>1-\frac{1}{\sigma_{\max }(\mathbf{A})},
$$

or

$Q^{-1}\left(\eta \alpha^{2} \mathbf{c b}\right) \sigma_{\min }\left(\mathbf{A}^{-1} \mathbf{b}\right) \sigma_{\min }\left(\mathbf{c}\left(\mathbf{A}-a_{1} \mathbf{I}_{n \times n}\right)\right)>1-\frac{1}{\sigma_{\max }(\mathbf{A})}$.

$$
\begin{aligned}
& \rho_{1}:=1-\frac{1}{\sigma_{\text {max }}(\mathbf{A})}, \\
& \rho_{2}:=\sigma_{\text {min }}\left(\mathbf{A}^{-1} \mathbf{b}\right), \\
& \rho_{3}:=\sigma_{\text {min }}\left(\mathbf{c}\left(\mathbf{A}-a_{1} \mathbf{I}_{n \times n}\right)\right),
\end{aligned}
$$

it gives

$$
Q^{-1} \eta \alpha^{2}>\frac{\rho_{1}}{\rho_{2} \rho_{3}} \text {. }
$$

Satisfying inequality (50) guarantees stability of the closed-loop system. In order to remove the steady-state error, the following relation must hold:

$$
\begin{aligned}
\lim _{k \rightarrow \infty} e(k+1)= & \lim _{k \rightarrow \infty} \mathbf{c}\left(\mathbf{A}-\mathbf{b} Q^{-1}(\eta \alpha \mathbf{c b})\left(\alpha \mathbf{c}\left(\mathbf{A}-a_{1} \mathbf{I}_{n \times n}\right)\right)\right) \mathbf{x}(k) \\
& +\lim _{k \rightarrow \infty} \mathbf{c b} Q^{-1}(\eta \alpha \mathbf{c b})\left(\alpha r_{d}(k+1)+a_{1} \alpha r_{d}(k)\right) \\
& -\lim _{k \rightarrow \infty} \mathbf{c b} Q^{-1}(\eta \alpha \mathbf{c b}) a_{2} \operatorname{sgn}(s(k)) \\
& +\lim _{k \rightarrow \infty} \mathbf{c b} Q^{-1} \psi(k)+\lim _{k \rightarrow \infty} \mathbf{c} \mathbf{w}(k)-\lim _{k \rightarrow \infty} r_{d}(k+1)=0 .
\end{aligned}
$$

Assuming $\quad \lim _{k \rightarrow \infty} \mathbf{x}(k)=\mathbf{x}_{\infty}, \quad \lim _{k \rightarrow \infty} r_{r}(k)=r_{d}$, $\lim _{k \rightarrow \infty} s(k)=0, \lim _{k \rightarrow \infty} \psi(k)=0$ and $|\mathbf{c w}(k)| \leq \Gamma_{w}$, it yields

$$
\begin{aligned}
\lim _{k \rightarrow \infty} e(k+1)= & \mathbf{c G} \mathbf{x}_{\infty}+\Gamma_{w} \\
& +\mathbf{c b} Q^{-1}(\eta \alpha \mathbf{c b})\left(\alpha\left(1+a_{1}\right) r_{d}-a_{2}\right)-r_{d}=0 .
\end{aligned}
$$

For satisfying (52), the following equations must be hold:

$$
\begin{aligned}
& \mathbf{c} \mathbf{G} \mathbf{x}_{\infty}+\mathbf{c b} Q^{-1}\left(\eta \alpha^{2} \mathbf{c b}\right)\left(1+a_{1}\right) r_{d}=r_{d}, \\
& \Gamma_{w}-\mathbf{c b} Q^{-1}(\eta \alpha \mathbf{c b}) a_{2}=0 .
\end{aligned}
$$

This completes the proof.

The block diagram of the proposed control method is shown in Fig. 2. The SMC law is determined in an optimal and recursive manner by realizing the reaching law, control limits, and minimizing the performance index.

By defining

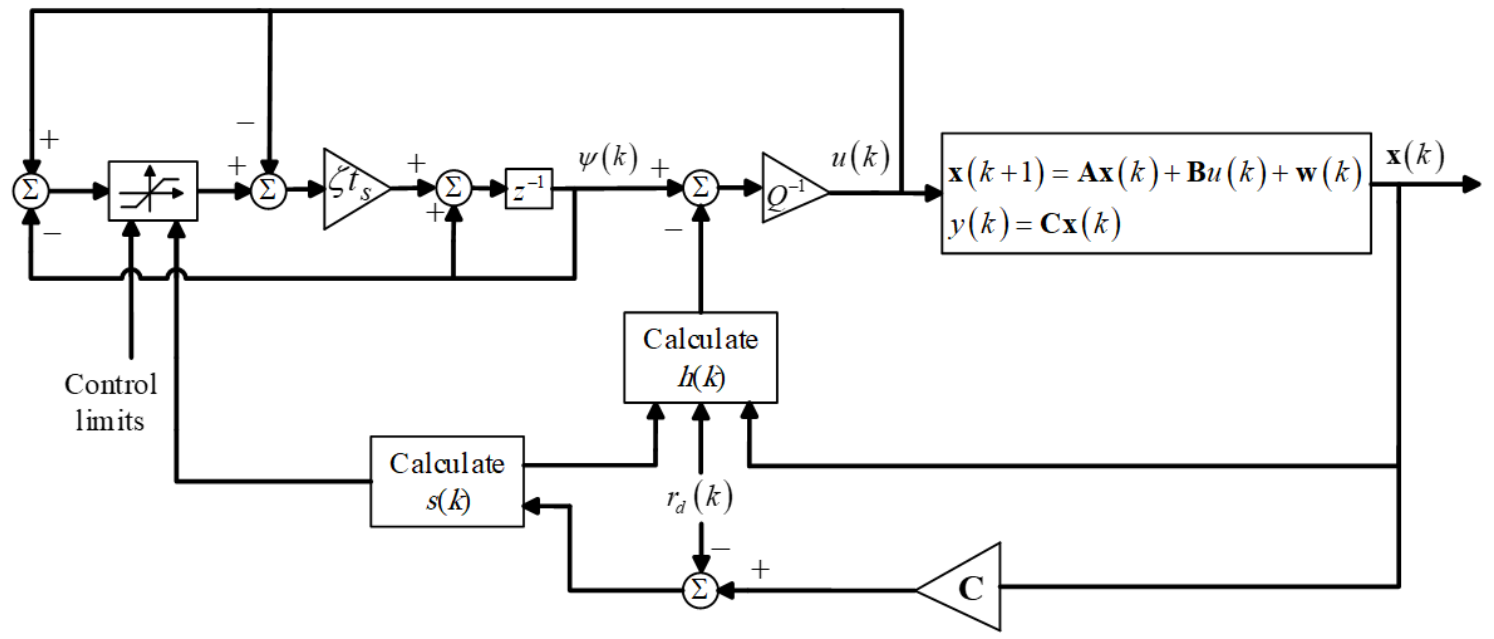

Fig. 2 Block diagram of the proposed discrete-time SMC based on PRNN 
Remark 4 Calculation of $\rho_{1}, \rho_{2}$ and $\rho_{3}$ in (50) depends on the system dynamics and parameter $\lambda_{1}$. Moreover, parameter $Q$ is related to parameters $\eta, \mu$ and $\alpha$. Therefore, these parameters have vital role in the stability of the closed-loop system in the presence of model uncertainties.

The procedure of determining the control parameters is given below

- Determine the sampling time $t_{s}$

- Choose relatively small positive value $\mu$

- Determine $\alpha, \eta$ and $\lambda_{1}$ for satisfying (53)

- Compute $a_{1}=\left(1-\lambda_{1} t_{s}\right)$

- Compute $\rho_{1}, \rho_{2}$ and $\rho_{3}$ in (49)

- Check (50) based on obtained $\alpha, \eta$ and $\lambda_{1}$

- Determine $\lambda_{2}$ using (54)

Remark 5 It should be noticed that no inversion of the input matrix is needed and hence, singularities are avoided.

In order to obtain the parameters so that the conditions (24) and (25) are satisfied, we have solved the following cost function:

$$
\begin{gathered}
\min _{Q, \alpha, \eta, \lambda_{1}, \lambda_{2}}\left(\mathbf{c G} \mathbf{x}_{\infty}+\mathbf{c b} Q^{-1}(\eta \alpha \mathbf{c b})\left(\alpha\left(a_{1}+1\right) r_{d}\right)-r_{d}\right)^{2} \\
+\left(\mathbf{c b} Q^{-1}(\eta \alpha \mathbf{c b}) a_{2}-\Gamma_{w}\right)^{2}
\end{gathered}
$$

subject to $Q^{-1} \eta \alpha^{2}(\mathbf{c b})>\frac{\rho_{1}}{\rho_{2} \rho_{3}}$

The above optimization problem should be solved offline and using several numerical optimization such as interior-point and active-set algorithms.

\section{Simulating example}

In order to evaluate performance of the proposed control method, consider the high precision position control of a piezomotor-driven linear stage system with the following state equations (Du et al. 2016):

$$
\begin{aligned}
& \&=x_{2} \\
& \&=\frac{k_{f}}{m} u-\frac{k_{v}}{m} x_{2}+f,
\end{aligned}
$$

where $x_{1}$ and $x_{2}$ denote linear displacement and linear velocity of the piezo motor shaft, respectively, and $u$ is the input voltage. The model uncertainty function $f$ is considered as $f(t)=0.2+0.1 \sin (0.5 \pi t)$.

The parameters of the model are $k_{f}=6, k_{v}=144$ and $m=1$. By selecting the sampling time $\left(t_{s}\right)$ equal to 0.01 second, the discrete-time model of the system can be expressed as follows:

$$
\begin{aligned}
& \mathbf{x}(k+1)=\left[\begin{array}{ll}
1 & 0.0053 \\
0 & 0.2396
\end{array}\right] \mathbf{x}(k)+\left[\begin{array}{c}
0.002 \\
0.0318
\end{array}\right] u+\mathbf{w}(k) \\
& y(k)=\left[\begin{array}{ll}
0.5 & 0.5
\end{array}\right] \mathbf{x}(k),
\end{aligned}
$$

where $\mathbf{w}(k)=\left[\begin{array}{ll}0 & t_{s} f(k)\end{array}\right]^{T}$. Using (50) and (53) for system in (57) yields $\rho_{1}=1.4875 \times 10^{-5}, \rho_{2}=0.1342$ and $\rho_{3}=0.2963$. Moreover, the designing parameters are obtained as $\eta=20, \mu=0.01$ and $\alpha=5$. The parameters of the sliding surface are selected as $\lambda_{1}=20$ and $\lambda_{2}=0.1$. Moreover, the learning rate of the PRNN is selected as $\zeta=0.1$. The control goals are stabilizing and tracking the displacement and velocity of the piezo-motor shaft. The initial conditions of the state variables are selected as $\mathbf{x}(0)=\left[\begin{array}{ll}-1 & 0\end{array}\right]^{T}$. The control limit is considered as $|u| \leq 24 \mathrm{~V}$.

As shown in Fig. 3, displacement of the piezo motor shaft converges to zero for less than 6 seconds. There is no overshoot with high precision steady-state response. Moreover, the proposed algorithm has successfully coped with the effects of model uncertainty. In addition, there is no chattering in the state variables.

According to Fig. 4, no chattering exists in the control signal, and it is within the predefined range. Moreover, the sliding surface gradually converges to zero. As shown in Fig. 6, the reaching law in (4) gradually converges to zero. This means that the proposed algorithm is able to satisfy the principle condition to ensure closed-loop stability. Also, the performance index in (9) that converges to zero relatively fast.

As shown in Fig 6, the computational time for each step of performing the proposed algorithm is less than one millisecond, which is far less than the sampling time.

It should be mentioned that $\lambda_{2}$ is obtained based on (54), while $\lambda_{1}$ must be chosen such that (50) and (53) are satisfied. Moreover, by selecting $\lambda_{1}$ ? $\lambda_{2}$, complete elimination of chattering is obtained. Therefore, there must be a tradeoff between the elimination of chattering and closed-loop stability for selecting $\lambda_{1}$.

Finally, the tracking problem for $y_{d}=1$ is considered. For comparison, the simulation results of the conventional SMC are presented. Based on typical reaching law, the SMC law for the dynamics model in (1) can be formulated as follows:

$$
u(k)=\frac{1}{\alpha \mathbf{c b}}\left[-\alpha \mathbf{c A x}+\alpha r_{d}(k+1)+s(k)-t_{s} \lambda_{2} \operatorname{sign}(s(k))\right] .
$$

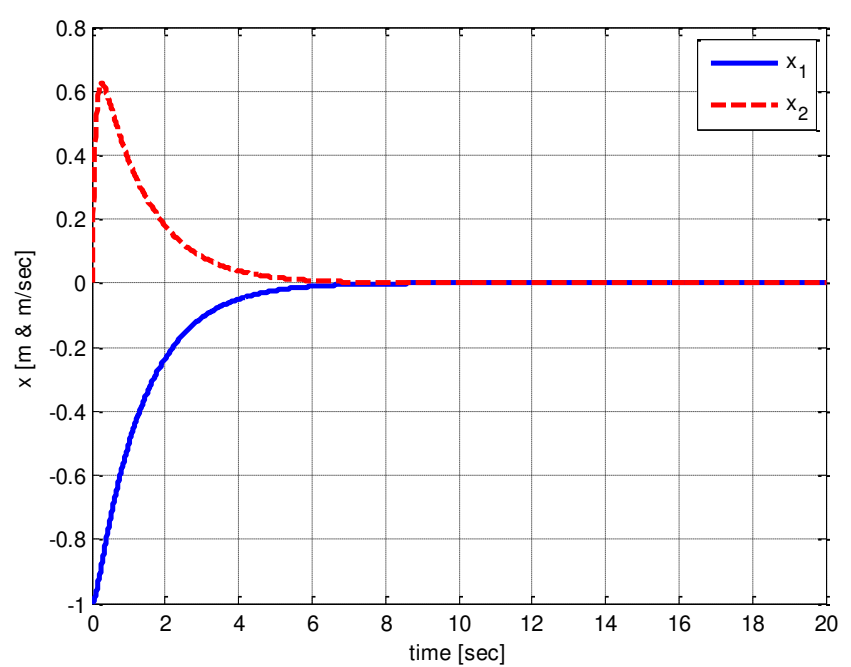

Fig. 3 Position and velocity of piezo motor using PRNN-based SMC 

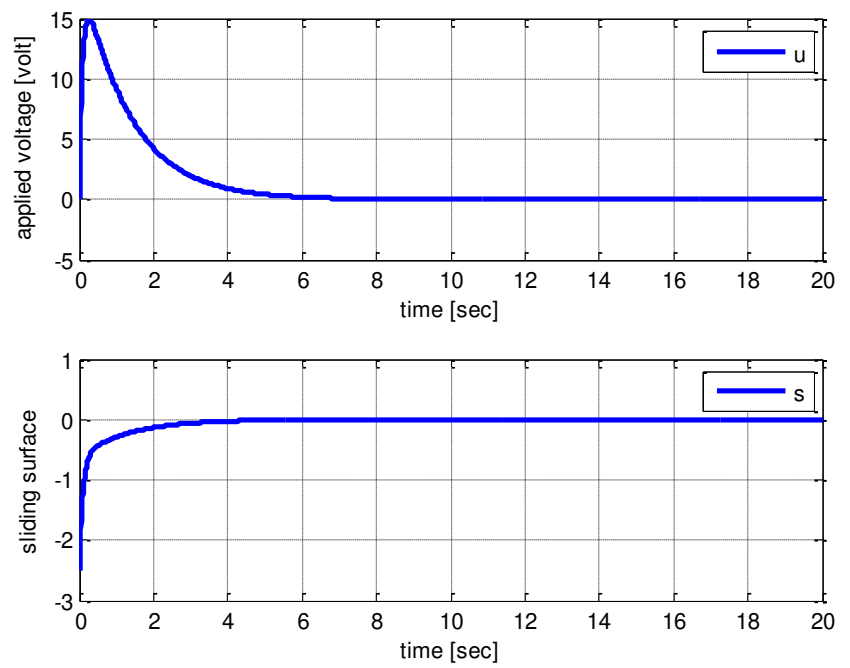

Fig. 4 Input voltage and sliding surface of PRNN-based DSMC
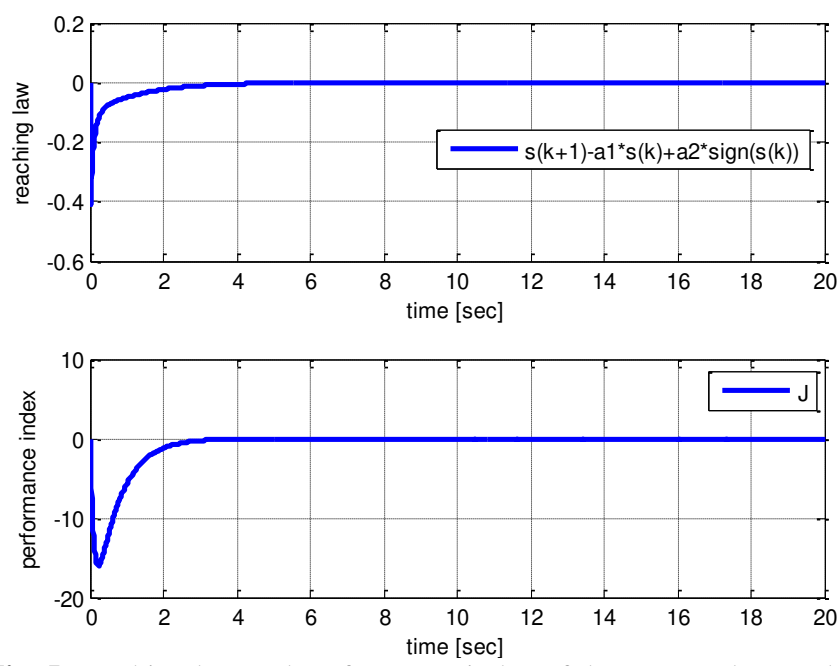

Fig. 5 Reaching law and performance index of the proposed control algorithm

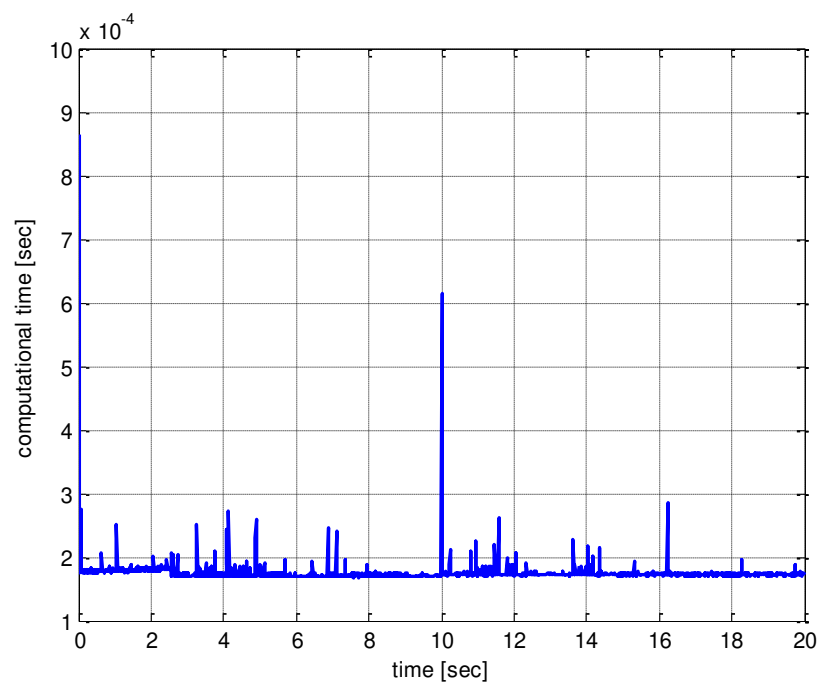

Fig. 6 Computational time for each step of performing the PRNNbased DSMC
Moreover, the performance of the proposed method is also compared with the chattering-free discrete-time SMC in (Du et al. 2016), called non-smooth control-based DSMC. The proposed SMC law is as follows:

$$
\begin{aligned}
u(k)=- & (\mathbf{c K})^{-1}\left[\mathbf{c} \mathbf{\Phi}(k)-s(k)+q_{1} h_{s} s(k)\right] \\
& -(\mathbf{c K})^{-1}\left[q_{2} h_{s} \operatorname{sgn}(s(k))|s(k)|^{\alpha_{n}}+\mathbf{c} \hat{\mathbf{d}}(k)\right],
\end{aligned}
$$

where

$$
\begin{aligned}
& \mathbf{\Phi}=e^{\mathbf{A} . h_{s}}, \mathbf{K}=\int_{0}^{h_{s}} e^{\mathbf{A} \tau} \mathbf{b} d \tau, \\
& \hat{\mathbf{d}}(k)=\mathbf{x}(k)-\boldsymbol{\Phi} \mathbf{x}(k-1)-\mathbf{K} u(k-1), \\
& s(k)=\mathbf{c x}(k) .
\end{aligned}
$$

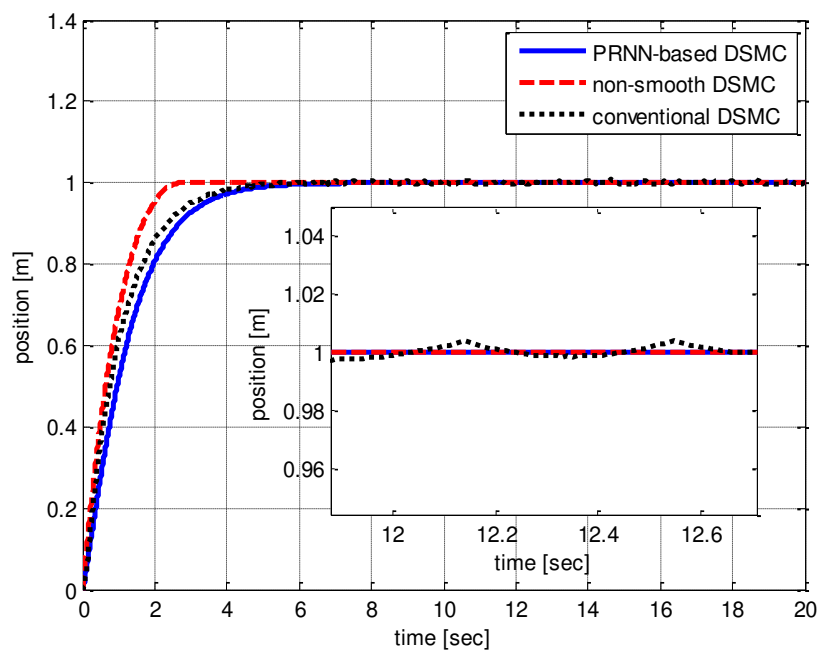

Fig. 7 Position of piezo motor using PRNN-based DSMC, nonsmooth DSMC and conventional DSMC

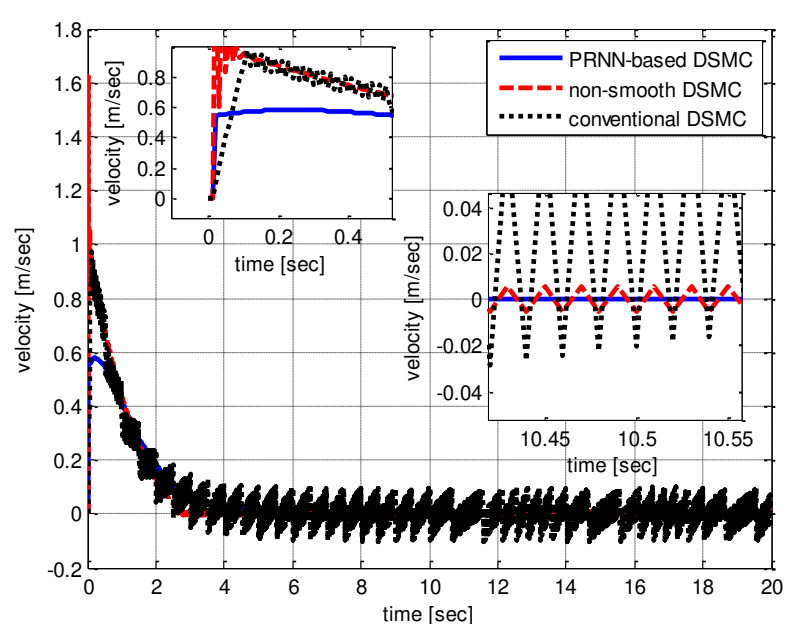

Fig. 8 Velocity of piezo motor using PRNN-based DSMC, nonsmooth DSMC and conventional DSMC 
The best performance for the conventional DSMC and nonsmooth DSMC are obtained with $\alpha=2, \lambda_{2}=1, q_{1}=q_{2}=3$, $\alpha=0.5$ and $h_{s}=0.01$, respectively.

As shown in Fig 7, all the proposed method, the non-smooth DSMC and conventional DSMC have good responses for position of the system. In other words, the displacement response of the piezo motor for all methods converges to desired value after six seconds. Although, some chattering around the desired position exists in response of the conventional method.

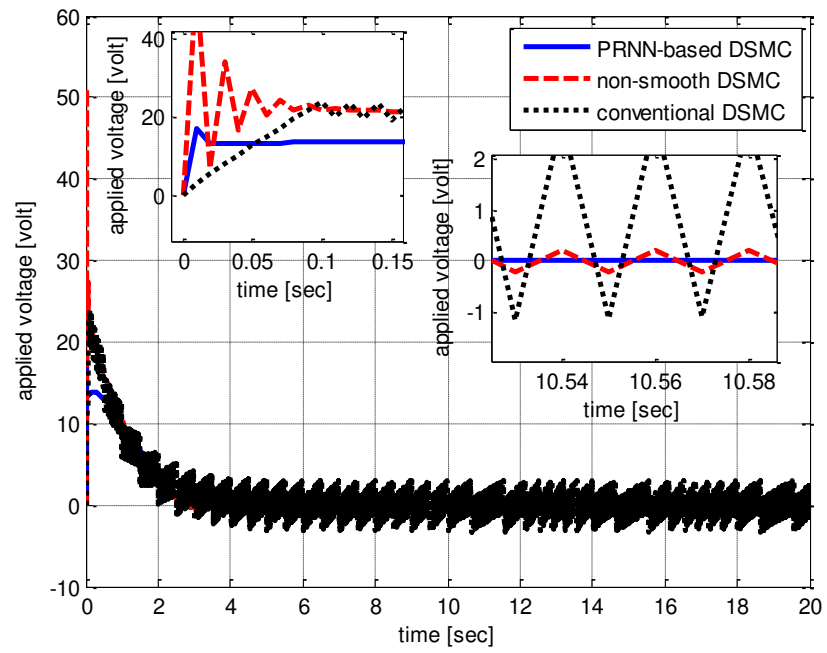

Fig. 9 Control signal obtained by PRNN-based DSMC, non-smooth DSMC and conventional DSMC
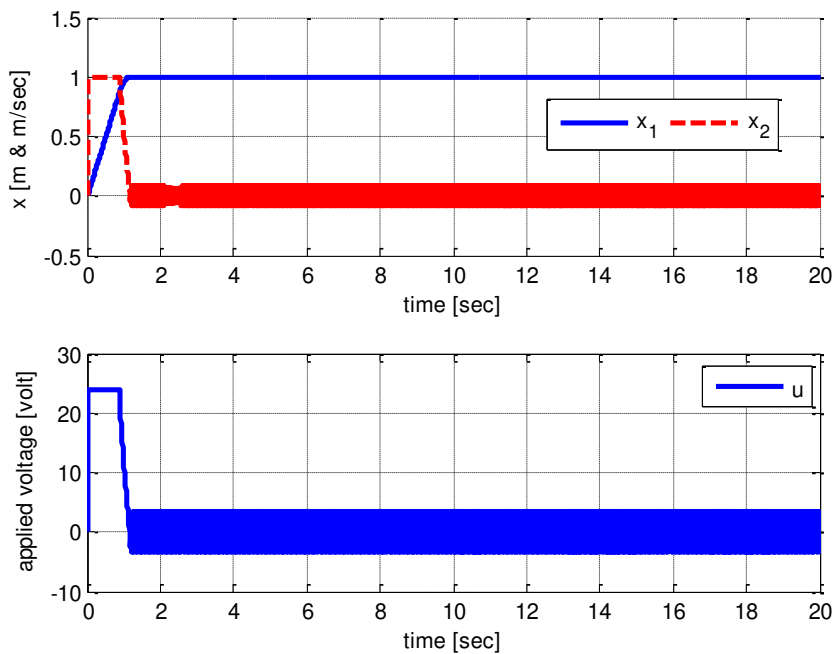

Fig. 10 Position, Velocity and voltage of piezo motor using the nonsmooth DSMC by imposing input saturation

According to Fig. 8, the velocity of the piezo motor for the proposed method converges smoothly to zero, while the nonsmooth DSMC has more variations through both transient and steady state conditions. The velocity response of the conventional DSMC has more sever changes respect to the other methods. Moreover, as Fig. 9 shows, the non-smooth DSMC violates the input voltage constraints at the beginning, and it is along with some chattering through steady state. In addition, the conventional DSMC has very weak performance with sever chattering in input signal.

As shown in Fig. 10, if a saturation is imposed to the control law of the non-smooth DSMC method (as it would in practice), the response is degraded drastically. In addition, the velocity response and control signal are varied severely. This is the main advantage of the proposed method as it can incorporate the constraints on the input signal in the optimization procedure.

Based on (5), parameter $\mu$ is used to minimize the control effort and to avoid numerical instability during online optimization procedure. Parameter $\eta$ has the main contribution to achieve the minimum tracking error. Moreover, parameter $\lambda_{2}$ is used to attenuate the effects of model uncertainties. Moreover, $\lambda_{2}$ is helpful to improve the transient responses and to eliminate the effects of chattering as well. In addition, $\eta$ and $\alpha$ contribute to the internal stability of the closed-loop system. In other words, larger values of $\eta$ provides more accurate responses, whilst by choosing larger values for $\mu$, less energy is consumed. Moreover, larger values for $\alpha$ can improve the tracking accuracy, but it may increase the closed-loop sensitivity to the model uncertainties.

\section{Conclusion}

In this paper, an optimal sliding-mode control method based on the projection recurrent neural networks was proposed for a class of discrete-time systems. To formulate the SMC law, a performance index including the minimum tracking error and the minimum control effort was defined and converted to a QP problem. The reaching law was involved in the cost function and the control limits were considered as the static constraints. For solving the constrained optimization problem, a discretetime projection-based neural network including a dynamic and an algebraic output equations were derived. It was shown that the proposed method was able to avoid chattering both in system responses as well as in control signal. Moreover, it was able to consider the constraints of the control signal. Moreover, it avoids singularity since there was no need for inversion of the input matrix. The control parameters were adjusted offline using the singular value approach.

\section{Declarations}

Funding: Not applicable

\section{Conflicts of interest/Competing interests}

We declared that we have no conflicts of interest to our entitled "Optimal discrete-time sliding-mode control based on recurrent neural network and: a singular value approach".

Availability of data and material: Not applicable

Code availability: Not applicable

Authors' contributions: Not applicable 


\section{References}

Amini MR, Shahbakhti M, Pan S, Hedrick JK (2017) Bridging the gap between designed and implemented controllers via adaptive robust discrete sliding mode control. Control Eng Pract, 59:1-15

Argha A, Li L, Su SW, Nguyen H (2016) On LMI-based sliding mode control for uncertain discrete-time systems. J Franklin Inst 353(15):3857-3875

Bartoszewicz, A (1998) Discrete-time quasi-sliding-mode control strategies. IEEE Trans Ind Electron 45(4):633-637

Chakrabarty S, Bartoszewicz A (2016) Improved robustness and performance of discrete time sliding mode control systems. ISA Trans 65:143-149

Chakrabarty S, Bandyopadhyay B (2015) A generalized reaching law for discrete time sliding mode control. Automatica 52:83-86

Du H, Yu X, Chen MZ, Li S (2016) Chattering-free discrete-time sliding mode control. Automatica 68:87-91

Feng J, Qin S, Shi F, Zhao X (2017) A recurrent neural network with finite-time convergence for convex quadratic bilevel programming problems. Neural Comput Appl 30(11):3399-3408

Furuta K (1990) Sliding mode control of a discrete system. Syst Control Lett 14(2):145-152

Gao W, Wang Y, Homaifa A (1995) Discrete-time variable structure control systems. IEEE Trans Ind Electron 42(2):117-122

Golo G, Milosavljević Č (2000) Robust discrete-time chattering free sliding mode control. Syst Control Lett 41(1):19-28

Huber O, Acary V, Brogliato B, Plestan F (2014) Discrete-time twisting controller without numerical chattering: analysis and experimental results with an implicit method. 53rd IEEE Annual Conference on Decision and Control (CDC), Los Angeles, CA, USA

Kinderlehrer D, Stampacchia G (1980) An introduction to variational inequalities and their applications. Siam, Philadelphia, PA, USA

Lee PM, Hong SW, Lim YK, Lee CM, Jeon BH, Park JW (1999) Discrete-time quasi-sliding mode control of an autonomous underwater vehicle. IEEE J Ocean Eng 24(3):388-395

Liu Q, Dang C, Cao J (2010) A novel recurrent neural network with one neuron and finite-time convergence for k-winners-take-all operation. IEEE Trans Neural Netw 21(7):1140-1148

Liu Q, Wang J (2013) A one-layer projection neural network for nonsmooth optimization subject to linear equalities and bound constraints. IEEE Trans Neural Netw Learn Syst 24(5):812-824

Liu S, Wang J (2006) A simplified dual neural network for quadratic programming with its KWTA application. IEEE Trans Neural Netw 17(6): 1500-1510

Liu Q, Wang J (2015) A projection neural network for constrained quadratic minimax optimization. IEEE Trans. IEEE Trans Neural Netw Learn Syst 26(11):2891-2900

Ma H, Wu J, Xiong Z (2016) Discrete-time sliding-mode control with improved quasi-sliding-mode domain. IEEE Trans Ind Electron 63(10):6292-6304

Maciejowski JM (1989) Multivariable Feedback Design. Electronic Systems Engineering Series. Wokingham, England: AddisonWesley

Meyer CD (2000) Matrix analysis and applied linear algebra. Siam. Philadelphia, USA

Milosavljević Č, Draženović B, Veselić B (2016) Discrete-time sliding-mode servo systems design with disturbance compensation approach. Complex Syst 597-618. https://doi.org/10.1007/978-3-319-28860-4_29

Miao P, Shen Y, Xia X (2014) Finite time dual neural networks with a tunable activation function for solving quadratic programming problems and its application. Neurocomputing 14:80-89

Sharma NK, Janardhanan S (2017) Discrete higher order sliding mode: concept to validation. IET Control Theory Appl 11(8):1098-1103
Skogestad S, Postlethwaite I (2017) Multivariable feedback control: analysis and design. John Wiley and Sons, New York

Su X, Liu X, Shi P, Yang R (2017) Sliding mode control of discretetime switched systems with repeated scalar nonlinearities. IEEE Trans Autom Control 62(9):4604-4610

Xia Y, Wang J (1998) A general methodology for designing globally convergent optimization neural networks. IEEE Trans Neural Netw 9(6):1331-1343

Xia Y, Wang J (2005) A recurrent neural network for solving nonlinear convex programs subject to linear constraints. IEEE Trans Neural Netw 16(2):379-386

Xiong J, Gan Q, Ren W (2016) Boundedness of discretised non-linear systems under fast terminal sliding mode control. IET Control Theory Appl 10(16):2100-2109

Xu Q, Cao Z (2017) Piezoelectric positioning control with outputbased discrete-time terminal sliding mode control. IET Control Theory Appl 11(5):694-702

Yan Y (2014) A new nonlinear neural network for solving QP problems. 11th International Symposium on Neural Networks, Hong Kong and Macao, China

Yan JJ, Liao TL (2018) Discrete sliding mode control for hybrid synchronization of continuous Lorenz systems with matched/unmatched disturbances. Trans. Inst. Meas. Control, 40(5):1417-1424

Zhou H, Lao L, Chen Y, Yang H (2017) Discrete-time sliding mode control with an input filter for an electro-hydraulic actuator. IET Control Theory Appl 11(9):1333-1340

\section{Appendix}

In this appendix, the stability of the projection recurrent neural network presented in (18) and (19) is investigated. Consider the dynamic equation of the PRNN as follows:

$\psi(k+1)=\psi(k)+\Theta(k)$,

where

$$
\Theta(k)=\zeta T\left(\operatorname{Pr}_{\Omega}\{u(k)-\psi(k)\}-u(k)\right) .
$$

Consider the following Lyapunov function:

$v(k)=\left(\psi(k)-\psi^{*}\right)^{T} \Lambda\left(\psi(k)-\psi^{*}\right)$,

where $\Lambda=1+Q^{-1}$. Based on (60), it can be written

$$
\begin{aligned}
v(k+1)= & \left(\psi(k+1)-\psi^{*}\right)^{T} \Lambda\left(\psi(k+1)-\psi^{*}\right) \\
= & \left(\psi(k)-\psi^{*}+\Theta(k)\right)^{T} \Lambda\left(\psi(k)-\psi^{*}+\Theta(k)\right) \\
= & \left(\psi(k)-\psi^{*}\right)^{T} \Lambda\left(\psi(k)-\psi^{*}\right)+\left(\psi(k)-\psi^{*}\right)^{T} \Theta(k) \\
& +\left(\psi(k)-\psi^{*}\right)^{T} Q^{-1} \Theta(k)+\Theta^{T}(k) \Theta(k) \\
& +\Theta^{T}(k) Q^{-1} \Theta(k)+\Theta^{T}(k)\left(\psi(k)-\psi^{*}\right) \\
& +\Theta^{T}(k) Q^{-1}\left(\psi(k)-\psi^{*}\right) .
\end{aligned}
$$

The first difference of $v(k)$ is equal to 


$$
\begin{aligned}
v(k+1)-v(k)= & \left(\psi(k)-\psi^{*}\right)^{T} \Theta(k)+\Theta^{T}(k) \Theta(k) \\
& +\left(\psi(k)-\psi^{*}\right)^{T} Q^{-1} \Theta(k) \\
& +\Theta^{T}(k) Q^{-1} \Theta(k)+\Theta^{T}(k)\left(\psi(k)-\psi^{*}\right) \\
& +\Theta^{T}(k) Q^{-1}\left(\psi(k)-\psi^{*}\right) .
\end{aligned}
$$

The following inequalities hold for any $\Theta \in \Omega$ (Liu and Wang 2006 ):

$$
\left(\psi(k)-\psi^{*}\right)^{T} \Theta(k)+\Theta^{T}(k) Q^{-1}\left(\psi(k)-\psi^{*}\right) \leq 0,
$$

$$
\left(\Theta(k)+Q^{-1}\left(\psi(k)-\psi^{*}\right)\right)^{T}\left(\psi(k)-\psi^{*}+\Theta(k)\right) \leq 0 .
$$

Using (63) and (64) and rearranging (62), it can be written as

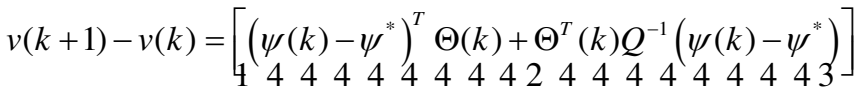

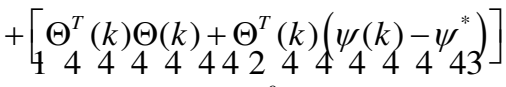

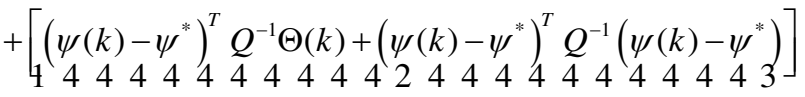

$$
\begin{aligned}
& +\left[\Theta^{T}(k) Q^{-1} \Theta(k)-\left(\psi(k)-\psi^{*}\right)^{T} Q^{-1}\left(\psi(k)-\psi^{*}\right)\right] .
\end{aligned}
$$

Hence, in order to ensure stability of the PRNN, it suffices that the following inequality holds:

$$
\Theta^{T}(k) Q^{-1} \Theta(k)-\left(\psi(k)-\psi^{*}\right)^{T} Q^{-1}\left(\psi(k)-\psi^{*}\right) \leq 0 .
$$

Based on (18) and (19), (67) can be reformulated as follows: $(\psi(k+1)-\psi(k))^{T} Q^{-1}(\psi(k+1)-\psi(k))$

$$
-\left(\psi(k)-\psi^{*}\right)^{T} Q^{-1}\left(\psi(k)-\psi^{*}\right) \leq 0 .
$$

Assuming that $|\psi(k+1)-\psi(k)| \leq \kappa\left|\psi(k)-\psi^{*}\right|$, where $\kappa$ is an arbitrary positive scalar, $v(k+1)-v(k) \leq 0$, which means that the PRNN is stable. 